\title{
Editorial: Interstitial Lung Disease in the Context of Systemic Disease: Pathophysiology, Treatment and Outcomes
}

\author{
Peter Korsten ${ }^{1 *}$, Maximilian F. Konig ${ }^{2}$, Björn Tampe ${ }^{1}$ and Mehdi Mirsaeidi ${ }^{3}$ \\ ${ }^{1}$ Department of Nephrology and Rheumatology, University Medical Center Göttingen, Göttingen, Germany, ${ }^{2}$ Division of \\ Rheumatology, Department of Medicine, The Johns Hopkins University School of Medicine, Baltimore, MD, United States, \\ ${ }^{3}$ Division of Pulmonary, Critical Care, and Sleep Medicine, University of Miami Miller School of Medicine, Miami, FL, \\ United States
}

Keywords: interstitial lung disease, autoimmune diseases, rheumatoid arthritis, systemic sclerosis (scleroderma), myositis

Editorial on the Research Topic

Interstitial Lung Disease in the Context of Systemic Disease: Pathophysiology, Treatment and Outcomes

\section{INTRODUCTION}

Interstitial lung disease (ILD) is an umbrella term for many different disease entities causing inflammation and fibrosis of the lung parenchyma. These can be broadly divided into five categories

OPEN ACCESS

Edited and reviewed by:

Laurent Pierre Nicod,

University of Lausanne, Switzerland

${ }^{*}$ Correspondence:

Peter Korsten

peter.korsten@med.uni-goettingen.de

Specialty section:

This article was submitted to

Pulmonary Medicine,

a section of the journal

Frontiers in Medicine

Received: 19 December 2020 Accepted: 24 December 2020

Published: 20 January 2021

Citation:

Korsten P, Konig MF, Tampe B and Mirsaeidi M (2021) Editorial: Interstitial

Lung Disease in the Context of

Systemic Disease: Pathophysiology,

Treatment and Outcomes.

Front. Med. 7:644075.

doi: 10.3389/fmed.2020.644075 based on etiology (1): (1) ILD related to a distinct primary disease (e.g., sarcoidosis), (2) ILD related to environmental factors (e. g., hypersensitivity pneumonitis), (3) ILD induced by drugs or irradiation, (4) idiopathic interstitial pneumonias (e.g., idiopathic pulmonary fibrosis), and (5) ILD related to connective tissue diseases (CTD) (1). While all these entities require thorough and multidisciplinary assessment to ascertain a diagnosis, establish the need for diagnostic procedures, and recommend a patient-specific treatment plan, ILDs associated with systemic diseases are particularly challenging. In many cases, optimal treatment for involvement of other organ systems needs to be balanced with the choice of ILD-directed therapies.

For the highly heterogeneous group of patients who cannot be given a definite diagnosis of an autoimmune rheumatic disease but who demonstrate certain clinical, radiographic, and/or serological features suggestive of a CTD, the term interstitial pneumonia with autoimmune features (IPAF) has been coined (2), but its clinical value remains to be defined. Due to its complexity, management of ILDs associated with systemic disease requires multidisciplinary care, including pulmonologists, rheumatologists, and radiologists, often with critical input from other specialties, such as pathologists, dermatologists, or neurologists. In this Research Topic, we hope to present novel research and state-of-the-art reviews as relevant to the care of patients with ILD and systemic diseases.

\section{FREQUENTLY ENCOUNTERED ILDS ASSOCIATED WITH SYSTEMIC DISEASES}

The most frequently encountered ILDs in the setting of systemic diseases include parenchymal lung involvement with myositis (Myo-ILD), systemic sclerosis (SSc-ILD), and rheumatoid arthritis 


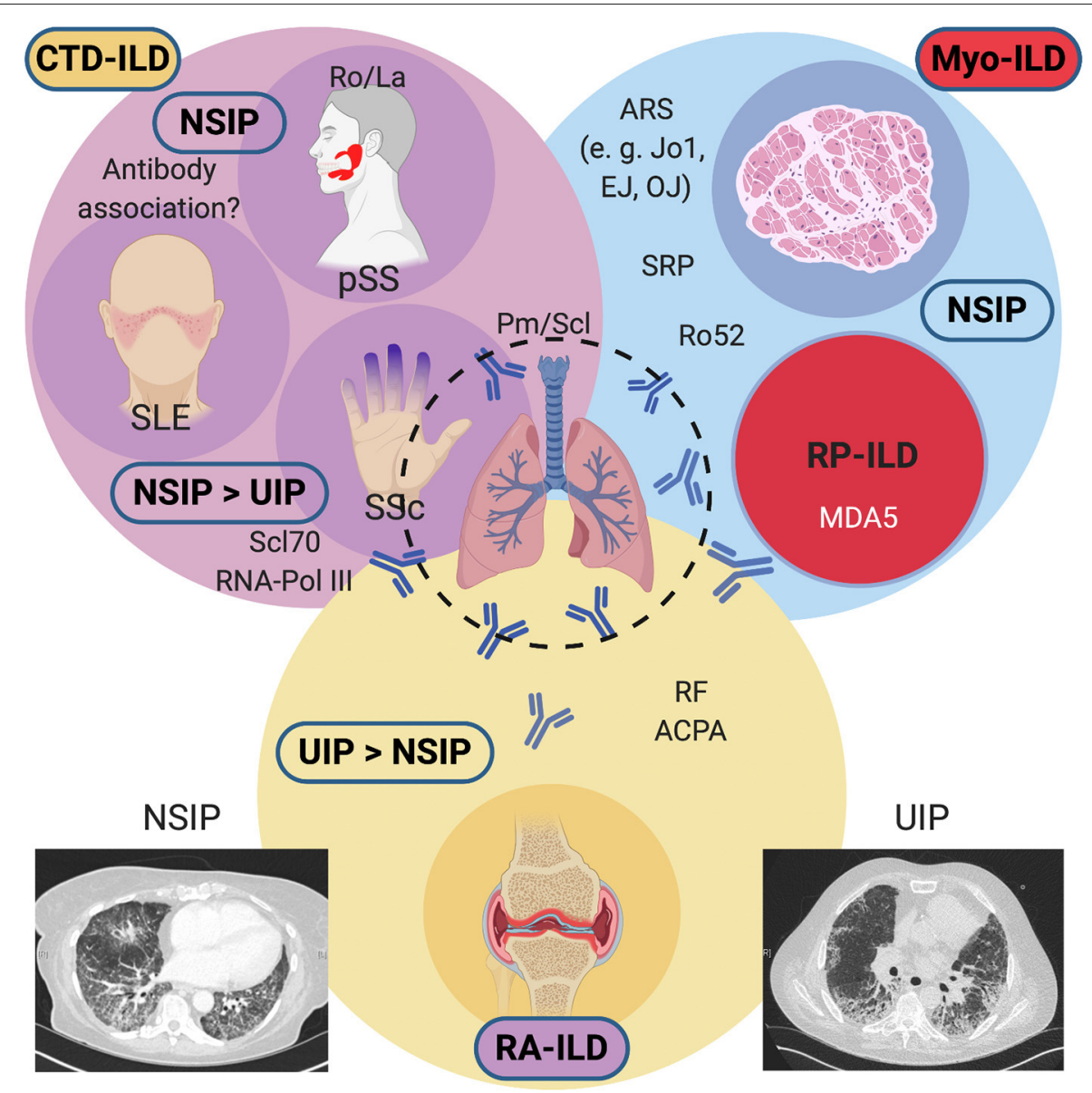

FIGURE 1 | Overview of systemic diseases causing interstitial lung diseases (ILDs) and their associated antibodies. Conditions associated with a predominant NSIP pattern on HRCTs include Myositis-associated ILD (antisynthetase syndrome, anti-RO52 positive overlap myositis, or RP-ILD), Systemic Sclerosis, primary Sjögren's syndrome, and rarely, Systemic Lupus Erythematosus. In SSc, a UIP pattern may also be commonly found. RA-ILD frequently shows a UIP pattern and confers a guarded prognosis. An NSIP pattern may also be encountered on HRCTs. ACPA, anti-citrullinated peptide antibodies; ARS, aminoacyl-tRNA synthetase antibodies; CTD, connective tissue disease; HRCT, high-resolution computed tomography; MDA5; melanocyte-differentiation antigen 5; Myo, myositis; NSIP, non-specific interstitial pneumonia; Pm/Scl, polymyositis-scleroderma; pSS; primary Sjögren's syndrome; RA, rheumatoid arthritis; RF, rheumatoid factor; RNA Pol III, RNA polymerase III; RP, rapidly progressive; Scl70, topoisomerase I; SLE, systemic lupus erythematosus, Sm, Smith; SRP, signal recognition particle; SSc, systemic sclerosis; U1-snRNP, U1 small nuclear ribonucleoprotein; UIP, usual interstitial pneumonia. Created with BioRender.com.

(RA-ILD). Less frequently reported are ILDs secondary to primary Sjögren's syndrome (pSS) or systemic lupus erythematosus (SLE). Figure 1 gives an overview of systemic diseases and frequently encountered autoantibodies that have been associated with the development of ILD in these diseases. For practical reasons, we find it helpful to distinguish these based on their predominant pattern on high-resolution computed tomography (HRCT), most frequently usual interstitial pneumonia (UIP) and non-specific interstitial pneumonia (NSIP). UIP changes tend to be less reversible and tend to confer a worse prognosis, whereas changes in NSIP (particularly in patients with non-fibrotic NSIP) may be more responsive to treatment. In different diseases, these lung disease patterns occur with varying frequency. In RA, UIP is often more frequently encountered than NSIP, although both may occur. Anti-cyclic citrullinated peptide (CCP) antibodies and rheumatoid factor
(RF), present in $70-80 \%$ of patients with RA, are associated with RA-ILD. The same applies to SSc, where NSIP is common, but UIP also occurs. In pSS, pulmonary manifestations are relatively rare but have been reported to occur in about $16 \%$ of patients, and a consensus guideline on the diagnostic and therapeutic approach has recently been published (3). In pSS, the occurrence of a lymphocytic interstitial pneumonitis (LIP) is rare, but this radiologic pattern should prompt a search for other manifestations of pSS if ILD is the first presenting manifestation (4). ILD is common in myositis, especially in patients with antibodies directed against different aminoacyltRNA synthetases, a group collectively called the antisynthetase syndrome (ASyS), but also those with anti-Ro52 antibodies $(5,6)$. Anti-MDA5 antibodies are associated with clinically amyopathic dermatomyositis (CADM) which can present as a rapidly progressive ILD with high mortality despite aggressive 
immunosuppressive therapy (7). The following summarizes the papers publishes within this Research Topic.

\section{A MULTIDISCIPLINARY APPROACH IS USEFUL FOR THE DIAGNOSIS AND MANAGEMENT}

In the first paper, Furini et al. systematically investigated the evidence for multidisciplinary conferences (MDC) to assess ILDs. They found that most MDC evaluated patients with history taking and clinical assessment, HRCT, pulmonary function tests (PFTs), lung biopsy (in most MDCs), and serological testing. Less consensus existed on the use of additional tests, such as nailfold video capillaroscopy (NVC) and 6-min walking distance. Only seven studies evaluated the rheumatologist's role in MDCs, but the authors suggest that MDCs include serological testing and rheumatology expertise to help classify CTD-ILD and IPAF with more certainty. The second paper by Tirelli et al. reported results from a retrospective cohort study from Pavia, Italy. In their cohort, the authors found that $15 \%$ of all patients were diagnosed with CTD-ILD, 33\% were classified as IPAF; the remainder had no underlying systemic disease. They found the application of a standardized screening questionnaire, laboratory testing, and the inclusion of NVC useful for detecting these entities. Karampitsakos et al. summarized the current use and ongoing clinical trials of biological therapies in sarcoidosis and idiopathic pulmonary fibrosis, which emphasizes a potentially useful role of rheumatologists in the management of ILDs given their extensive expertise in the use of these drugs and management of complications.

\section{CONNECTIVE TISSUE DISEASE-ASSOCIATED ILD}

One paper specifically reported findings in SLE, pSS, and SSc patients. Patients with SLE and pSS tend to have less ILD. Therefore, it is even more critical to identify individuals at risk and potential overlap syndromes among patients with these relatively common autoimmune rheumatic diseases. Amarnani et al. reviewed the pulmonary manifestations of SLE, including ILDs. Interestingly, the development of an ILD in SLE has not been associated with anti-dsDNA antibodies but rather anti-Ro or anti-U1-snRNP antibodies, which are also associated with mixed connective tissue disease (MCTD). It is, therefore, unclear whether these patients instead represent an overlap population. Sogkas et al. reported their singlecenter experience from a large cohort of pSS patients with ILD. At their center, $13 \%$ of patients were eventually diagnosed with ILD. Of note, almost two thirds (61\%) were diagnosed

\section{REFERENCES}

1. Wijsenbeek M, Cottin V. Spectrum of Fibrotic Lung Diseases. N Engl J Med. (2020) 383:958-68. doi: 10.1056/NEJMra2005230 with ILD at presentation and, surprisingly, UIP was the most commonly encountered pattern on HRCT (43\%). Lastly, Mirsaeidi et al. reviewed the current treatment options for SSc-ILD, an emerging and rapidly changing topic with the recently approved anti-fibrotic nintedanib, and several ongoing clinical trials.

\section{MYOSITIS-ASSOCIATED ILD}

The majority of papers addressed Myo-ILD. The review by Hervier and Uzunhan represents a timely and current overview of the diagnostic and therapeutic approach to Myo-ILD. This is expanded by original data from Asian cohorts on rapidlyprogressive ILD ( $\mathrm{Li}$ et al.), acute exacerbations of ILD in Myo-ILD (Liang et al.), and the understudied role of plasma exchange in the treatment of refractory Myo-ILD (Ning et al.). Finally, Korsten et al. report their findings on the efficacy of immunosuppression in ASyS-ILD. They specifically report equal usefulness of rituximab (RTX) compared to other conventional immunosuppressive drugs, especially in patients with more frequent clinical myositis and progressive or relapsing pulmonary involvement.

\section{RHEUMATOID ARTHRITIS-ASSOCIATED ILD}

The recent observation of $M U C 5 B$ promoter variants as a risk factor for RA-ILD, similar to patients with idiopathic pulmonary fibrosis, has generated significant interest in understanding the potential role of antifibrotic strategies in the treatment of this subset of ILD (8). Emerging data from cohort studies examining different immunosuppressive drugs [e.g., abatacept or RTX; $(9,10)$ ] is of similar interest. Fragoulis et al. provided an overview of the difficult topic of RA-ILD, which is an area of active investigation. The authors summarize data on methotrexate-induced pneumonitis and pulmonary fibrosis associated with RA.

\section{AUTHOR CONTRIBUTIONS}

PK wrote the first draft and created the figure. BT, MK, and MM edited and reviewed the draft. All authors approved the final version of the manuscript.

\section{ACKNOWLEDGMENTS}

We thank the reviewers and additional external editors for their time and thoroughness in assessing the submitted papers.

\footnotetext{
Fischer A, Antoniou KM, Brown KK, Cadranel J, Corte T), du Bois RM, et al. An official European Respiratory Society/American Thoracic Society research statement: interstitial pneumonia with autoimmune features. Eur Respir J. (2015) 46:976-87. doi: 10.1183/13993003.00150-2015
} 
3. Lee AS, Scofield RH, Hammitt KM, Gupta N, Thomas DE, Moua T, et al. Consensus guidelines for evaluation and management of pulmonary disease in Sjögren's. Chest. (2020). doi: 10.1016/j.chest.2020.10.011

4. Flament T, Bigot A, Chaigne B, Henique H, Diot E, Marchand-Adam S. Pulmonary manifestations of Sjögren's syndrome. Eur Respir Rev. (2016) 25:110-23. doi: 10.1183/16000617.0011-2016

5. Ferreira JP, Almeida I, Marinho A, Cerveira C, Vasconcelos C. Anti-ro52 antibodies and interstitial lung disease in connective tissue diseases excluding scleroderma. ISRN Rheumatol. (2012) 2012:415272. doi: 10.5402/2012/415272

6. Xing X, Li A, Li C. Anti-Ro52 antibody is an independent risk factor for interstitial lung disease in dermatomyositis. Respir Med. (2020) 172:106134. doi: 10.1016/j.rmed.2020.106134

7. Chino H, Sekine A, Baba T, Kitamura H, Iwasawa T, Okudela K, et al. Interstitial lung disease with anti-melanoma differentiationassociated protein 5 antibody: rapidly progressive perilobular opacity. Intern Med. (2019) 58:2605-13. doi: 10.2169/internalmedicine.23 28-18

8. Juge P-A, Lee JS, Ebstein E, Furukawa H, Dobrinskikh E, Gazal S, et al. MUC5B promoter variant and rheumatoid arthritis with interstitial lung disease. $N$ Engl J Med. (2018) 379:2209-19. doi: 10.1056/NEJMoa180 1562
9. Vadillo C, Nieto MA, Romero-Bueno F, Leon L, Sanchez-Pernaute $\mathrm{O}$, Rodriguez-Nieto MJ, et al. Efficacy of rituximab in slowing down progression of rheumatoid arthritis-related interstitial lung disease: data from the NEREA Registry. Rheumatology. (2020) 59:2099-108. doi: 10.1093/rheumatology/kez673

10. Fernández-Díaz C, Loricera J, Castañeda S, López-Mejías R, Ojeda-García C, Olivé $\mathrm{A}$, et al. Abatacept in patients with rheumatoid arthritis and interstitial lung disease: a national multicenter study of 63 patients. Semin Arthritis Rheum. (2018) 48:22-7. doi: 10.1016/j.semarthrit.2017.12.012

Conflict of Interest: The authors declare that the research was conducted in the absence of any commercial or financial relationships that could be construed as a potential conflict of interest.

Copyright $\odot 2021$ Korsten, Konig, Tampe and Mirsaeidi. This is an open-access article distributed under the terms of the Creative Commons Attribution License (CC $B Y)$. The use, distribution or reproduction in other forums is permitted, provided the original author(s) and the copyright owner(s) are credited and that the original publication in this journal is cited, in accordance with accepted academic practice. No use, distribution or reproduction is permitted which does not comply with these terms. 